\title{
Pulsed energy storage antennas for ionospheric modification
}

\author{
R. F. Wuerker ${ }^{1, *}$, J. Chen ${ }^{1}$, W. Beam ${ }^{2}$, and E. Nichols ${ }^{2}$ \\ ${ }^{1}$ UCLA Plasma Physics Lab., Los Angeles, CA 90095, USA \\ ${ }^{2}$ HIPAS Observatory Two Rivers, AK 99712, USA \\ *now retired from UCLA
}

Received: 3 November 2003 - Revised: 4 May 2004 - Accepted: 18 May 2004 - Published: 31 January 2005

Part of Special Issue "Eleventh International EISCAT Workshop"

\begin{abstract}
Interesting, "new", very high peak-power pulsed radio frequency (RF) antennas have been assembled at the HIPAS Observatory (Alaska, USA) and also at the University of California at Los Angeles (UCLA, USA); namely, a pair of quarter wavelength $(\lambda / 4)$ long cylindrical conductors separated by a high voltage spark gap. Such a combination can radiate multi-megawatt RF pulses whenever the spark gap fires. The antenna at HIPAS is $53 \mathrm{~m}$ long $(\lambda / 2)$ with a central pressurized $\mathrm{SF}_{6}$ spark gap. It is mounted 5 meters $(\lambda / 21)$ above a ground plane. It radiates at $2.85 \mathrm{MHz}$. The two antenna halves are charged to \pm high voltages by a Tesla coil. Spark gap voltages of $0.4 \mathrm{MV}$ (at the instant of spark gap closure) give peak RF currents of $\sim 1200 \mathrm{~A}$ which correspond to $\sim 14 \mathrm{MW}$ peak total radiated power, or $\sim 56 \mathrm{MW}$ of Effective Radiated Power (ERP). The RF pulse train is initially square, decaying exponentially in time with Qs of $\sim 50$. Two similar but smaller $80-\mathrm{MHz}$ antennas were assembled at UCLA to demonstrate their synchronization with a pulsed laser which fired the spark gaps in the two antennas simultanoeously. These experiments show that one can anticipate a pulsed array of laser synchronized antennas having a coherent Effective Radiated Power (ERP) $>10 \mathrm{GW}$. One can even reconsider a pulse array radiating at $1.43 \mathrm{MHz}$ which corresponds to the electron gyrofrequency in the Earth's magnetic field at $\sim 200 \mathrm{~km}$ altitude. These "new" pulsed high power antennas are hauntingly similar to the ones used originally by Hertz (1857-1894) during his (1886-1889) seminal verifications of Maxwell's (1864) theory of electrodynamics.
\end{abstract}

Key words. Electromagnetics (antennas) - Ionosphere (polar ionosphere, active experiments) - Space plasma physics (changed particle motion and acceleration)

\section{Introduction}

Electromagnetic, or $\mathrm{E}$ and $\mathrm{M}$, waves at $\sim 50 \mathrm{MHz}$ were first generated in the year of 1886 when a pair of $30-\mathrm{cm}$ diameter conducting spheres $(\sim 15 \mathrm{pF}$ each) were interconnected

Correspondence to: R. F. Wuerker

(rwuerker@adelphia.net) by two one-meter long straight conductors $(\sim 1.5 \mu \mathrm{H}$ inductors), through a central spark gap (Bryant, 1988). The two insulated resonators were oppositely charged by a high voltage induction coil. When the spark gap fired, the system radiated powerfully enough to induce a faint spark in another gap in a distant resonant receiving loop (Bryant, 1998). With such crude equipment, H. Hertz (1857-1894) detected standing waves on open wire lines. His calculated $\mathrm{L}-\mathrm{C}$ antenna frequency $(\sim 50 \mathrm{MHz})$ and measured standing wavelength along a transmission line gave wave velocities close to the value predicted by J. Maxwell (1864); namely, $\mathrm{c}=1 / \sqrt{ } \varepsilon \mu$ (Buchwald, 1994), as well as the velocity of light values determined earlier by H. L. Fizeau (1849) and J. L. Foucault (1850) (Jenkins, 1957). Additional experiments by Hertz (1888) at $\sim 500 \mathrm{MHz}$ duplicated all the then known optical phenomena; namely, reflection of his waves from metals, focusing by parabolic reflectors, refraction, polarization, and interference. His experiments interconnected Maxwell's theory of electricity and magnetism with the optical region of the spectra.

Later, negative resistance plasma arcs in a transverse magnetic field (Poulson arcs) generated the sustained RF oscillations that launched practical RF communications. Arc generators were subsequently replaced by evacuated electron tubes that can generate multi-kilowatt transmissions. Arrays of coherently oscillating antennas are now used to achieve even higher concentrated ERPs. For example, the HIPAS CW Ionospheric heater in Alaska has eight bi-resonant crossed dipole antennas within a 210-m diameter circle (Wong et al., 1990). Each antenna is driven by an Eimac 4CV100 000 electron tetrode type of vacuum tube which can deliver $125 \mathrm{~kW}$ of continuous RF power. The ERP of the HIPAS array is $\cong 70 \mathrm{MW}$ at either 2.85 or $4.53 \mathrm{MHz}$. The continuously tunable HAARP facility, $285 \mathrm{~km}$ south of HIPAS, has a $6 \times 8$ antenna array within a $120 \times 150 \mathrm{~m}$ patch. This facility achieves $20 \mathrm{MW}$ ERP at $3 \mathrm{MHz}$ to $200 \mathrm{MW}$ ERP at its upper frequency of $8.2 \mathrm{MHz}$ (Kennedy, 2002). Both facilities generate similar levels of ELF within the polar electro jet by amplitude modulation of their carrier frequency (Milikh, 2002). Both facilities also produce similar sky glows or artificial auroral patches when RF power is directed along the earth's 


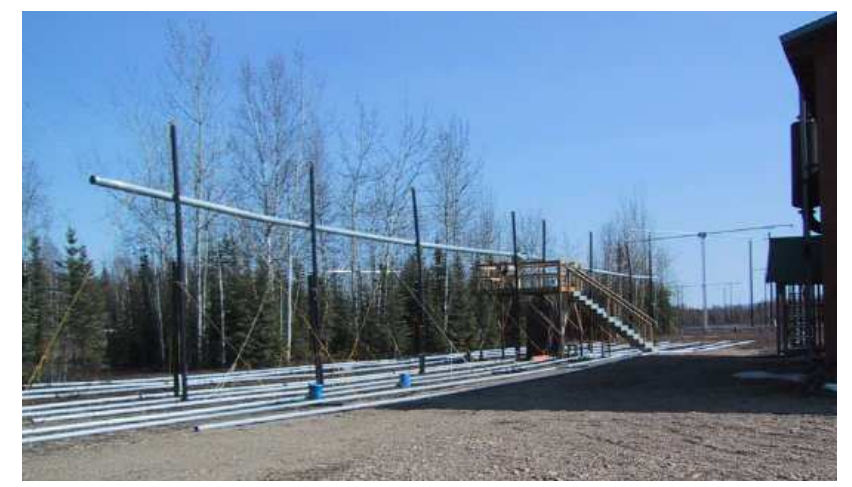

Fig. 1. Photograph (April 2003) of the 2.85-MHz (18-cm diameter $-5200 \mathrm{~cm}$ long) Hertzian antenna at HIPAS, suspended from insulating 10-cm diameter ABS drainpipes, $500 \mathrm{~cm}$ above a ground plane made up of $6.4-\mathrm{cm}$ diameter aluminum pipes. The antenna has a capacitance of $200 \mathrm{pF}$ across its central spark gap. The stairways are for central access. One of the antennas of the CW array at HIPAS can be seen in the background. The north side of the Generator Building is on the right side of the picture.

magnetic field (Sentman, 2002). HAARP is now doubling the size of its array to 192 antennas, 3.6 MW total radiated power, with expected ERP's of $400 \mathrm{MW}$ at $2.8 \mathrm{MHz}$ and $3 \mathrm{GW}$ at $10 \mathrm{MHz}$ (Kennedy, 2002)! Their upgrade is expected to be finished by 2006 (Kennedy, 2003).

Last year, the HIPAS group was asked to consider the implementation of an inexpensive pulsed RF system that would match the ERP of the new GW-HAARP array. Preliminary analysis dismissed pulsed electron tubes as too costly, even though HIPAS already had two 3 MW Machlett ML-7560 triodes. Pulsed $~ 30$ MW RF "frozen wave transmission lines" were considered next (Weibel, 1964) (Liette, 1965) (Stephan, Hugrass, and Jones, 1982). A twenty cycle L-C line, consisting of $6050-\mathrm{kV}-8 \mathrm{nF}$ ( $\$ 250$ each) capacitors, was designed. This approach was abandoned in favor of the simpler "frozen voltage" generator used originally by Hertz; namely, a pair of oppositely charged high voltage quarter wave long antennas separated by a spark gap. This simple radiator became more attractive after it was found that antenna $Q s \geq 10$ could be realized with $\mathrm{SF}_{6}$ filled spark gaps. It was also learned, for antenna heights $<\lambda / 4$ above a conducting ground, that the radiation pattern is unchanged while the antenna's radiation resistance varies linearly from $0-70 \Omega$ with height (Hall, 1984). Since the radiation resistance controls the antenna's $\mathrm{Q}$, antennas closer to ground are suddenly favored. As a result, antennas operating at the electron gyrofrequency in the ionosphere $(\sim 1.43 \mathrm{MHz})$ are no longer the difficult civil engineering challenge of conventional electron tube driven antennas at $\lambda / 5-\lambda / 4(50 \mathrm{~m})$ heights above ground.

A 2.85-MHz Hertzian or "energy storage pulsed RF system" has been assembled at the HIPAS Observatory from $18 \mathrm{~cm}$ outside diameter agricultural aluminum tubing. Each $\lambda / 4$ radiating element is $26 \mathrm{~m}$ long for a $\lambda / 2$ length of $52 \mathrm{~m}$. Originally, the two elements were separated by an open-air spark gap, which has since been replaced by one pressurized with $\mathrm{SF}_{6}$ gas. Across the spark gap, the antenna halves have a measured capacitance of $200 \mathrm{pF}$ or stored energy of $\sim 0.36$ Joules (J) when charged to $\pm 30 \mathrm{kV}$, giving $120 \mathrm{KW}$ of radiated power for $\mathrm{a} \sim 3 \mu$ s oscillation duration ( $\mathrm{Q} \sim 30$ ). If one could charge this antenna to 3 million Volts DC ( $3 \mathrm{MV}$, now used on some European open air power lines), one could expect a 300 MW RF pulse! Several such antennas working coherently together should approach ERPs of $\sim 10 \mathrm{GW}^{1}$ !

\section{The $2.85 \mathrm{MHz}$ hertzian antenna at HIPAS}

Starting last summer (2002), as noted above, a series of 2.8$\mathrm{MHz}$ Hertzian antennas were assembled and tested at the HIPAS Observatory. The first antennas were close to the ground, had open-air, self triggering spark gaps, different ground plane configurations, and were resistively charged by available $0-30 \mathrm{kV}$ supplies.

Concurrently, a smaller 80-MHz antenna was assembled at UCLA. Like Hertz' originals, this antenna had a very low Q due to its smaller stored energy and the higher resistance of its open-air spark gap. Replacement of the spark gap by one filled with pressurized $\mathrm{SF}_{6}$ reduced the gap spacing while greatly improving the antennas' $\mathrm{Q}$. This discovery resulted in the immediate installation of an $\mathrm{SF}_{6}$ gap on the HIPAS antenna, similarly improving its Q.

Figure 1 is a photograph of the $2.8-\mathrm{MHz}$ antenna at HIPAS, taken April 2003. As noted earlier, each 18-cm outside diameter (aluminum irrigation pipe) antenna half is $26 \mathrm{~m}$ long for a total antenna length of $52 \mathrm{~m}$. The whole antenna was hung $5 \mathrm{~m}$ above its ground plane by guyed $10-\mathrm{cm}$ diameter non-conducting polyvinyl chloride (ABS) drainpipes now commonly used in home plumbing. The ground plane (prominent in the picture) was made from smaller diameter $(6.4 \mathrm{~cm})$ aluminum agriculture pipes.

The stairways, seen in Fig. 1, are for easy access to the central spark gap and charging circuitry, shown in Fig. 2; namely, a picture of a long pressurized spark gap within the four fiberglass tie rods that bind the gap and antenna halves together. This photograph also shows the Tesla type of high voltage charging system, ${ }^{2}$ along with the current monitoring

\footnotetext{
${ }^{1}$ Unpublished Russian work; "Development of a Powerful Short Wave System," (1988-1989). This document describes a pulsed $17 \mathrm{MHz}$ system based on two $\pm 0.5 \mathrm{MV}$ quarter wave coaxial elements also separated by a pressurized spark gap, inside a shielded oil filled container. The RF power generated by their shielded resonator was capacitively coupled to a transmission line that drove an external antenna. This paper claimed RF outputs of $1.2 \mathrm{GW}$, when the internal resonator was charge to $3 \mathrm{KJ}$ of stored energy. This system was described by Sergey Rolyakov and Dimitry Kotik at the Polar Aeronomy and Radio Science (PARS) Conference, UCLA Conference Center, Lake Arrowhead, California, 3-6 November, 2002. Copies of the paper (in Russian) were sent to UCLA after the meeting.

${ }^{2}$ Soon to be replaced by an even larger coil system with a 33$\mathrm{cm}$ diameter - 354-cm long secondary of 1400 turns $(53.3-\mathrm{mH}$ and $600-\mathrm{pF}$ self capacitance) and two layer pancake primary of 14 turns having $166 \mu \mathrm{H}$ inductance.
} 


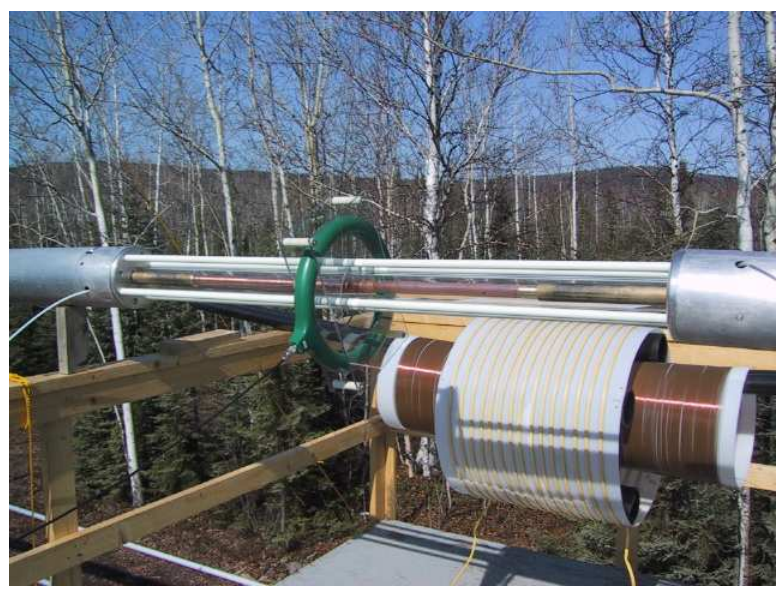

Fig. 2. Center of the 2.85-MHz Hertz dipole, seen in Fig. 1, showing the self firing pressurized $\mathrm{SF}_{6}$ spark gap, Tesla primary and secondary antenna charging coils, and green antenna current monitoring transformer, surrounding the electrodes of the spark gap.

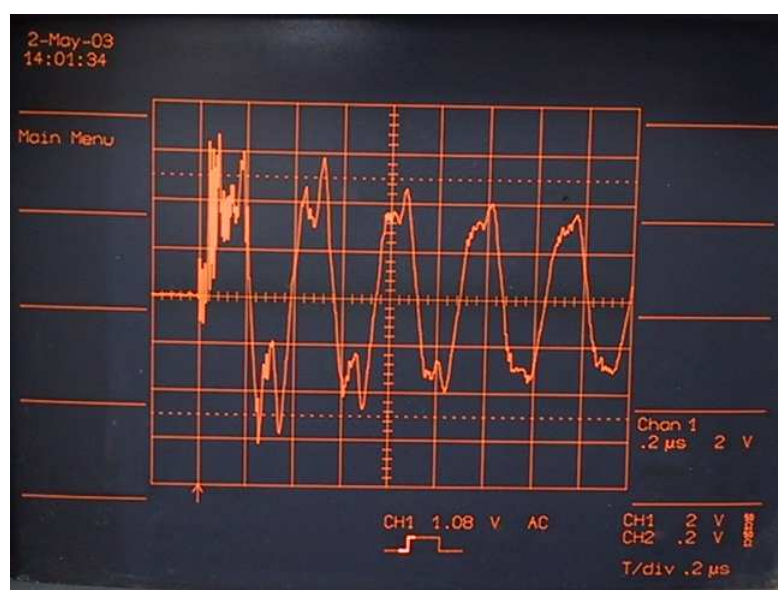

Fig. 3. Initial current pattern generated by the 2.85-MHz Hertzian antenna, seen in Fig. 1, as measured through a 10:1 voltage attenuator with the 0.05 V/A monitor, seen in Fig. 2. Each major vertical division corresponds to $400 \mathrm{~A}$. Each major horizontal division is $0.2 \mu \mathrm{s}$.

transformer surrounding the spark gap electrodes. Figure 3 shows a typical oscilloscope recording of the output of the current transformer through a 10:1 voltage attenuator; namely, instantaneous currents of $\sim 1200$, a peak which correspond to instantaneous radiated powers $\sim 11 \mathrm{MW}_{\text {rms }}$, assuming $9.3 \Omega$ of radiation resistance (independently measured with an RF bridge). Note the pronounced third harmonic of the antenna's fundamental frequency, a result of the initial DC square wave voltage on the antenna halves, before the spark gap fired. Figure 4 is another recording, taken at a slower oscilloscope sweep speed, showing the favorable $\mathrm{Q} \sim 50$ of the antenna, which can also be used to determine the reactive impedance of the dipole remembering that for a simple LC discharge circuit $\omega \mathrm{L} / \mathrm{R}=\mathrm{Q}$ with $\mathrm{Q}=\pi \mathrm{N}_{\text {eth }}$, where $\mathrm{N}_{\text {eth }}$ is the number of cycles to the $\exp (-1)$ or $37 \%$ amplitude

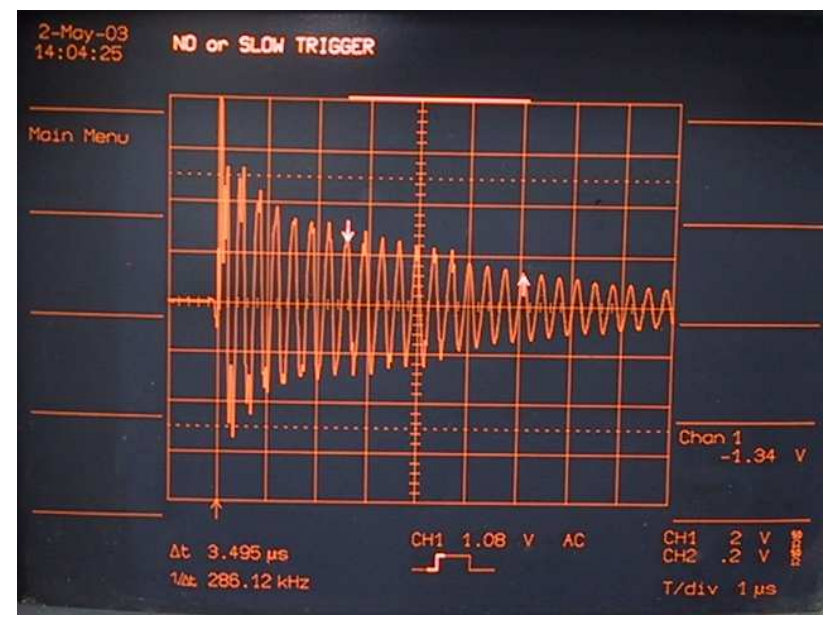

Fig. 4. Longer duration ( $1 \mu \mathrm{s} /$ major horizontal division) recording of the HIPAS antenna current, illustrating the favorable $Q \cong 50$ of these Hertzian antennas with $\mathrm{SF}_{6}$ spark gaps.

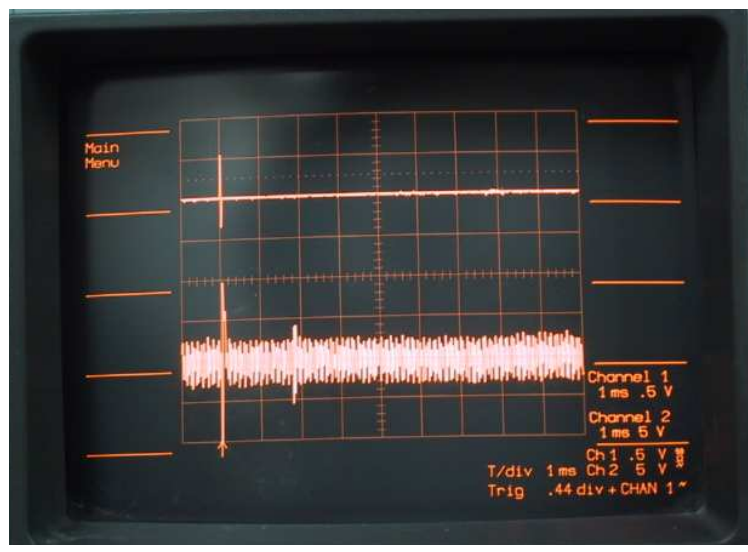

Fig. 5. Echoes from the ionosphere due to the pulse antenna seen in Fig. 1. The upper trace is the signal from the current monitor. The lower trace is the signal from a remote receiving (ionosonde) antenna at HIPAS showing an echo $1.9 \mathrm{~ms}$ later, corresponding to a reflection from a critical layer at $280 \mathrm{~km}$. In this case the RF pulse was only $9.3 \mathrm{~kW}$.

point. Figure 4 shows a $\mathrm{Q}$ of 47 which means that the two reactive impedances of the dipole at $2.85 \mathrm{MHz}$ were $\pm \mathrm{j} 422 \Omega$. For any high $\mathrm{Q}$ system, the initial DC voltage $\left(\mathrm{V}_{i}\right)$ on the capacitance, along with the reactive impedance of the circuit, determines the peak current during the first half cycle: $\mathrm{I}_{i}$ (at time $=1 / 4 \mathrm{f})=\mathrm{V}_{i} / \omega \mathrm{L}=\omega \mathrm{CV}_{i}=\mathrm{V}_{i} / \sqrt{ } \mathrm{L} / \mathrm{C}$, indicating that for the Fig. 4 example the initial voltage across the spark gap (when the spark gap connected the two antenna halves together) was $\cong 0.42 \mathrm{MV}$ or $\pm 210 \mathrm{KV}$.

Returns from the ionosphere due to the pulsed antenna have also been seen, as illustrated in Fig. 5 (recorded on 9 December 2002). This example shows the output of the current monitor on the pulsed antenna (upper trace, current peaks of 32 A corresponding to RF peaks of $9.3 \mathrm{~kW}$ ) and (lower trace) the return from a remote ionosonde antenna at 


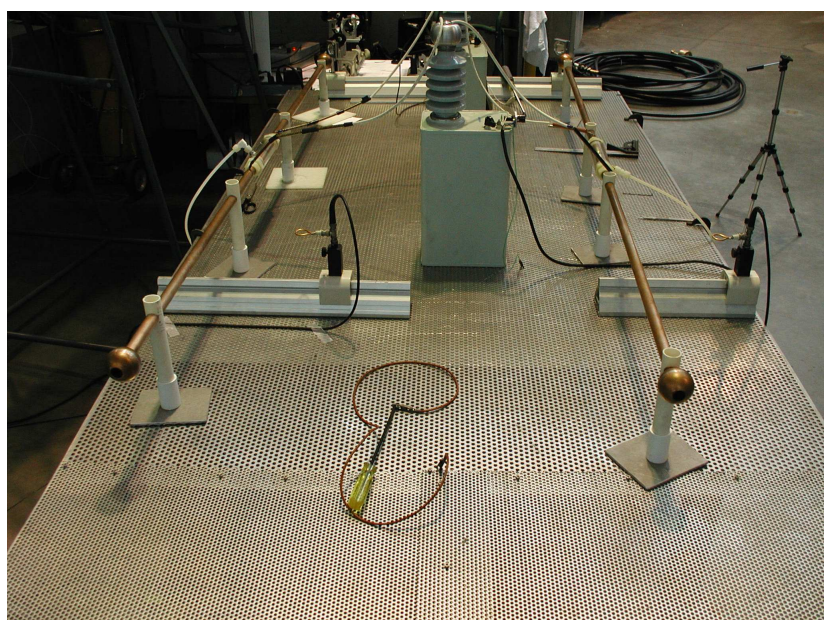

Fig. 6. Photograph of a pair of $80-\mathrm{MHz}$ laser triggered (via pressurized $\mathrm{SF}_{6}$ spark gaps) Hertzian antennas set up at UCLA, over a perforated aluminum ground plane. Each $\lambda / 4$ antenna half is $93 \mathrm{~cm}$ long and is mounted $17 \mathrm{~cm}$ above ground. The $\pm 0-75-\mathrm{kV}$ charging power supplies are between the two antennas. The laser beam splitters are in the background. Single turn loops for monitoring the antenna currents are mounted on the triangular optical rails. The laser beams pass into the antennas through holes in the high voltage ends, traveling inside the hollow conductors to a lens (which also serves as a high pressure spark gap window) which focuses the laser pulse, through a hole in the near electrode, onto a heavy metal (W-Cu) insert in the far electrode. The focused laser energy generates ultraviolet light that ionizes the $\mathrm{SF}_{6}$ gas in the two gaps.

HIPAS, which detected the reflection from the ionosphere $1.9 \mathrm{~ms}$ later, corresponding to a critical layer at $280 \mathrm{~km}$ altitude. At this same time, one antenna, on the HIPAS CW array, was pulsed at $10 \mathrm{~kW}$, resulting in an almost identical (equal in amplitude and equal in delay time) return from the ionosphere. False returns have also been seen when the spark gap was found to recharge, double pulsing the antenna with delays similar to what one would expect from the ionosphere.

\section{$2.180-\mathrm{MHz}$ Hertzian antennas at UCLA}

As noted earlier, a higher frequency version of the HIPAS antenna was set up at UCLA where space is a premium. The UCLA antenna found the $\mathrm{Q}$ enhancing benefits of $\mathrm{SF}_{6}$ gas in the spark gap. This same antenna also demonstrated the decrease in the $\mathrm{Q}$ of the antenna, with height above a flat ground plane (Hall, 1984). Next, the spark gap was modified to be fired or switched by a nanosecond duration pulse from a solid-state laser; namely, a new spark gap with an internal lens that would focus the laser pulse through a hole in one spark gap electrode onto the opposing electrode. Initial experiments, with a small $0.1 \mathrm{~J} /$ pulse $-15 \mathrm{~ns}$ YAG $(1060 \mathrm{~nm})$ pulsed solid-state laser, showed nanosecond delays between the irradiation of the spark gap and the onset of antenna oscillations. As a result of this favorable result, a second lasertriggered $80-\mathrm{MHz}$ antenna was assembled for coherent lasertriggering experiments. Initial experiments with the original
$0.1 \mathrm{~J} /$ pulse laser showed that after division of the laser pulse into two pulses, by a 50/50 beam splitter, the laser pulse energy was too low for jitter free switching. A more powerful larger laser, belonging to the UCLA Tokamak group, was made available to the authors; namely, a Doubled YAG laser of $0.7 \mathrm{~J} /$ pulse $-10-15 \mathrm{~ns}$ at $0.532 \mathrm{~nm}$. Figure 6 shows a picture of the dual antenna setup before the beam splitter arrangement that split the laser pulse which irradiated the spark gaps in the two antennas. Single turn loops, mounted on optical triangular rails (seen in Fig. 6), recorded the derivatives of the current in each antenna. The signals from the loops were monitored by a multi channel oscilloscope, which also monitored the output of the laser. Figure 7 shows the experimental setup schematically, while Fig. 8 shows the initial synchronization of two antennas to within nanoseconds. These experiments show that energy storage antennas can be made to emit coherently when triggered by a powerful enough laser pulse. In Fig. 8, the black trace is the derivative of the current from one antenna, while the violet trace is for the other antenna. The yellow trace is the laser pulse recorded with a vacuum photodiode. One can see that with time the two antennas were no longer synchronized due to slight length and resonant frequency differences. This problem was resolved by changing the length of one antenna a few millimeters $(\Delta \mathrm{f}=0.1 \mathrm{MHz})$. Figure 8 also shows that the $80 \mathrm{MHz}$ antennas had lower Qs $(\sim 10)$ than the $2.85-\mathrm{MHz}$ antenna at HIPAS (see Fig. 4). When the 80-MHz antenna was operated with an open-air spark gap its Q was even worse, $\sim 2$.

These experiments clearly showed that a coherent array of laser triggered pulsed Hertzian radiators could be assembled that can match or exceed the peak power of the new HAARP system for a fraction of its cost.

\subsection{Hertzian antenna properties}

It has been seen that the radiated output of a Hertz antenna is initially a damped square wave due to the spatial DC square wave on the antenna before the spark gap fires. The 3rd and 5 th harmonics of the antenna's fundamental frequency are basically further from the ground plane, with the result that they damp faster, leaving the antenna oscillating sinusoidally at its fundamental $(\lambda / 2)$ frequency. The first half cycle is the most powerful part of the pulse, unlike more conventional electron tube-driven (HIPAS, HAARP, and EISCAT) ionospheric heaters, whose outputs increase linearly with time; namely, with rise times of $\sim 10-100 \mu \mathrm{s}$. For the conventional case of a slowly rising RF square wave, nonresonant free electrons in the ionosphere end up vibrating out of phase with the electric vector of the radiation field, ending up with kinetic energies (in electron volts) of only;

$$
\begin{gathered}
E_{C W}(e V)=m v^{2} / 2=(1 / 2)(e / m)(E / \omega)^{2} \\
=(1 / 2)(e / m)\left(1 / \omega^{2}\right)\left(F Z_{o}\right),
\end{gathered}
$$

where $e / m$ is the electron charge-to-mass ratio $\left(1.79 \times 10^{11} \mathrm{C} / \mathrm{kgm}\right), E$ is the RF field strength (Volts $\left./ \mathrm{m}\right), \omega$ is the angular frequency of the RF field (radians/s) $F$ is the 


\section{The Laser Triggering Dipole Antenna Array $2 / 27 / 03$}

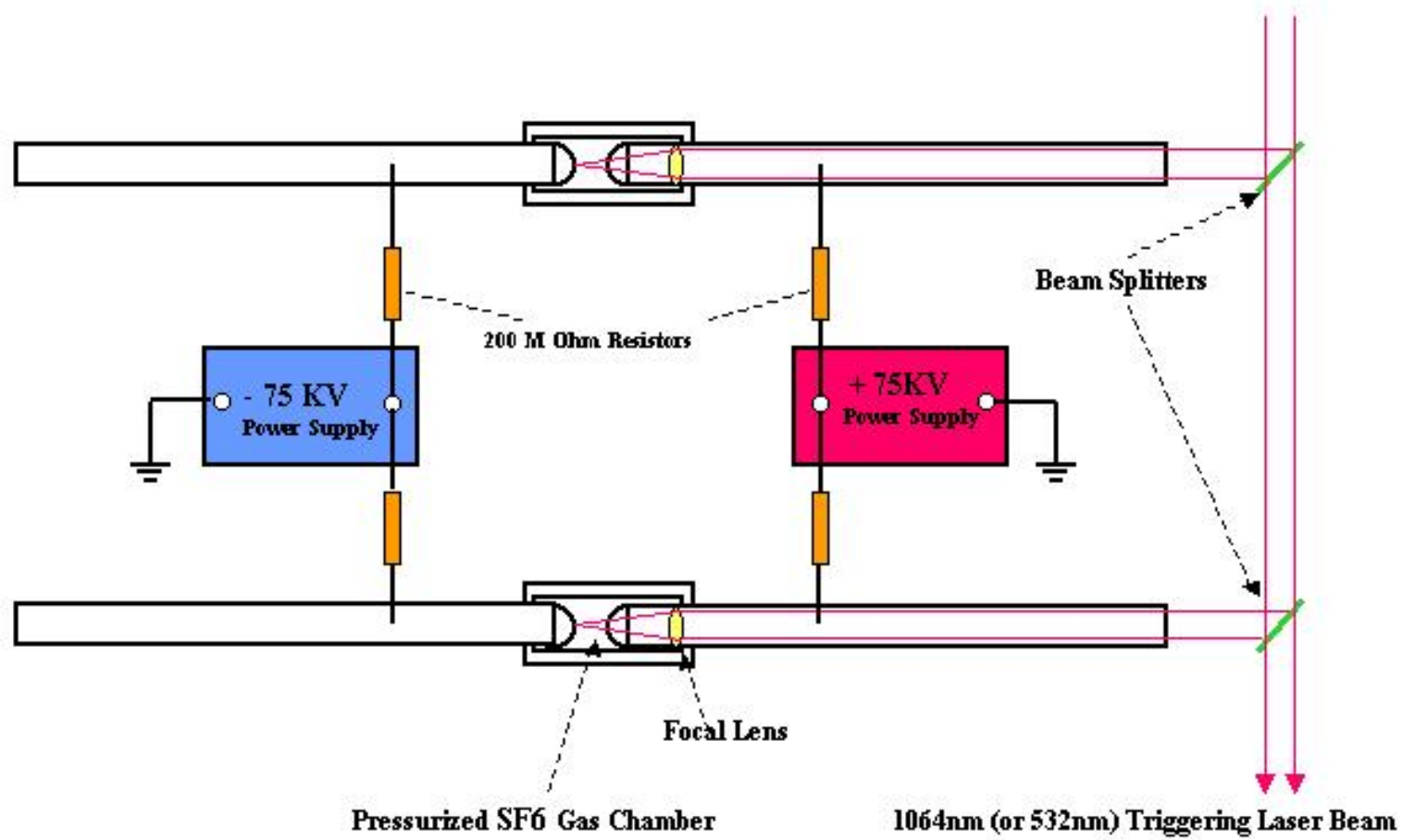

Fig. 7. Schematic of the dual $80-\mathrm{MHz}$ antenna laser triggering experiment.

\section{The Laser Synchronous Triggering of The Antenna Array 2/27/03}

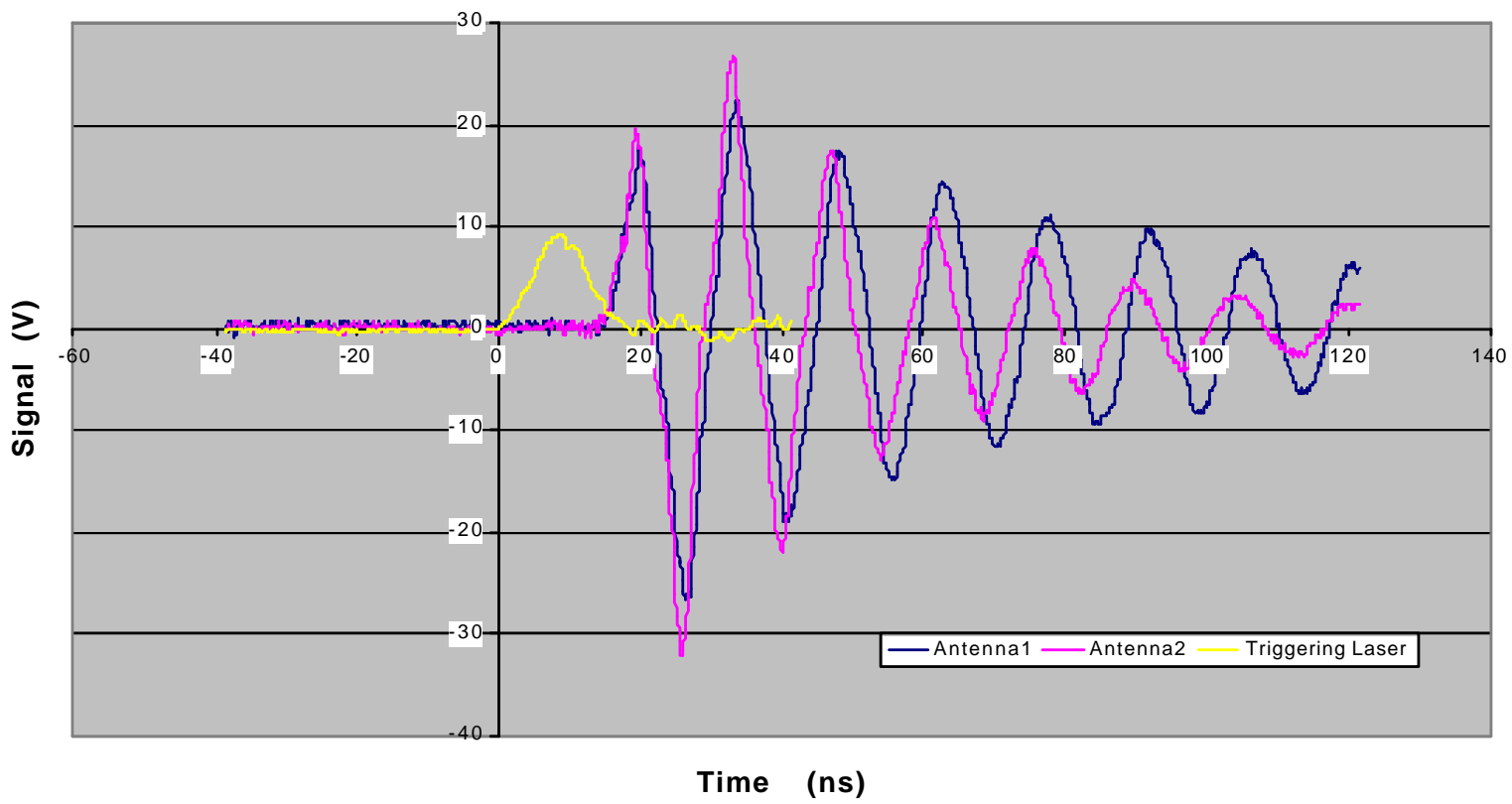

Fig. 8. Multi trace oscillogram showing the derivatives of the two $80-\mathrm{MHz}$ antenna currents (black and violet traces), triggered by the $0.5 \mathrm{~J}$ - 10-15 ns (532 nm) pulse (yellow trace) from a doubled YAG solid-state laser. 
Table 1. Calculated electron energies at 100-km altitude for pulsed Hertz antennas radiating at $1.43 \mathrm{MHz}$.

\begin{tabular}{|c|c|c|c|c|}
\hline Peak & Flux & Jitter & Impulse & $\begin{array}{l}\text { Resonance } \\
3 \text { RF cycles }\end{array}$ \\
\hline GW & & milli eV & milli eV & \\
\hline 0.05 & 0.4 & 0.14 & 1.5 & 0.06 \\
\hline 0.10 & 0.8 & 0.22 & 2.2 & 0.09 \\
\hline 0.20 & 1.6 & 0.24 & 2.4 & 0.1 \\
\hline 0.45 & 3.6 & 1.3 & 13 & 0.5 \\
\hline 0.80 & 6.4 & 2.3 & 22 & 0.9 \\
\hline 1.00 & 8.0 & 2.8 & 28 & 1.1 \\
\hline 1.25 & 10 & 3.5 & 35 & 1.4 \\
\hline 1.8 & 14.5 & 5.0 & 50 & 2.0 \\
\hline 2.45 & 19.5 & 7.0 & 70 & 2.8 \\
\hline 3.2 & 25.5 & 8.9 & 89 & 3.5 \\
\hline 4.05 & 32.5 & 11 & 115 & 4.4 \\
\hline 5.0 & 40 & 14 & 140 & 5.6 \\
\hline 10 & 80 & 28 & 280 & 11 \\
\hline
\end{tabular}

flux of the RF field $\left(\mathrm{W} / \mathrm{m}^{2}\right)$, and $\mathrm{Z}_{o}$ is the impedance of free space $(\sim 377 \Omega)$.

The first (square wave) half cycle from a Hertzian antenna accelerates free electrons uniformly, so that at the end of the initial half cycle an electron would have nearly an order of magnitude higher kinetic energy than its "jitter" in the nonresonant cyclical CW situation; namely,

$E_{\text {Hertz }}(e V)=\pi^{2}\left(E_{C W}\right)$.

However, in a non-scattering or collision free situation, energy acquired by an electron in the first half cycle of a square pulse would be extracted in the second half cycle, reaccelerated in the third half cycle, etc. Collisions, however, could result in the electron gaining energy on each half cycle.

Even more interesting is the case of true resonance where the electric vector of the radiation field is perpendicular to the Earth's magnetic field, with the electron oscillating at the cyclotron frequency. The electron cyclotron frequency at $200-\mathrm{km}$ altitude is $1.43 \mathrm{MHz}$, which indicates an antenna twice as long as the one shown in Fig. 1. At resonance a free electron would have an energy $\left(E_{\text {res }}\right)$ after $\mathrm{N}$ cycles of RF perpendicular to the Earth's magnetic field of

$E_{\text {res }}=4 \pi^{2} N^{2} E_{C W}$.

Only 3 full cycles of RF would increase the electron energy by a factor of $\sim 400$ over the non resonant jitter motion Eq. (1), making pulsed antennas operating at the electron cyclotron frequency particularly interesting.

Table 1 gives electron energy values at 100-km altitudes for different ERPs, for a system operating at $1.43 \mathrm{MHz}$, for the case of continuous RF, Eq. (1), at the end of the first cycle from a non-resonant Hertzian antenna, Eq. (2), and for the case of 3 cycles of cyclotron resonance, Eq. (3).

What is important is that ERPs of several GW at resonance will result in electron energies of several volts after only a few RF resonant cycles. Volts of energy are required for ionization of the background gas ( $\sim 2 \mu$ Torr at $200 \mathrm{~km}$ altitude).

\section{Summary}

A new class of high peak power radio frequency antennas for ionospheric modification applications is being developed at the HIPAS Observatory, Alaska. A pair of oppositely charged quarter-wave long radiators, separated by a high voltage spark gap, radiates at their common resonant frequency whenever the spark gap fires. Such an antenna can radiate $\sim 0.25 \mathrm{GW}$ pulses when the voltage across the spark gap is one million volts. The initial positive and negative charging of the antenna halves, puts a "frozen" square voltage wave onto the antenna. This same wave pattern is radiated when the spark gap fires; however, the exponentially damped square wave transforms into a sine wave due to the higher radiation resistance of the higher odd $3 \mathrm{rd}$, 5th, etc., harmonics of the antenna's fundamental frequency. It is predicted that this initially square wave output will impulsively accelerate electrons in the ionosphere. The duration of antenna oscillation is determined by the height of the antenna above ground plane, making possible ionospheric heating experiments at the electron cyclotron frequency in the earth's magnetic field (namely, $1.53 \mathrm{MHz}$ at $100 \mathrm{~km}$ altitude and $1.43 \mathrm{MHz}$ at $200 \mathrm{~km}$ ). Several antennas can be made to radiate coherently when triggered by a laser of sufficient pulse energy. As a result, pulsed ERPs in excess of one Gigawatt $\left(10^{9} \mathrm{~W}\right)$ and higher are suddenly possible at reasonable costs.

Acknowledgements. This project is under the direction of A. Wong who also reviewed it at the recent RF Ionospheric Interactions Workshop, Santa Fe, New Mexico, (Wong, 2003). The authors also wish to acknowledge the support of the HIPAS staff; R. Dickman (site manager), J. Foley, and E. Rodgers. We also thank R. Taylor (Director of the UCLA Tokamak Project) and Z. Lucky for our use of their $0.5 \mathrm{~J} /$ pulse $-5 \mathrm{~ns}$ (100 MW) Doubled YAG laser. Finally, we thank J. Movius of Fairbanks, Alaska, for his many array calculations and for recommending J. Buchwald's book (Buchwald, 1994).

Topical Editor M. Lester thanks S. J. Cowen for his help in evaluating this paper.

\section{References}

Bryant, J. H.: Heinrich Hertz, The Beginning of Microwaves, 1988 IEEE/MTT-S Hertz Centennial Celebration, IEEE, New York, N.Y. ISBN 0-87942-710-8, 1988.

Bryant, J. H.: Henrich Hertz's Experiments and Experimental Apparatus: His Discovery of Radio Waves and His Delineation of their Properties, in D. Baird, R. Hughes, and A. Nordman editors, Henrich Hertz: Classical Physicist, Modern Philosopher, Kluwer Academic Publishers, London, 39-58, 1998.

Buchwald, J. Z.: The Creation of Scientific Effects, H. Hertz and Electric Waves, University of Chicago Press, Chicago, Illinois, 1994. 
Hall, G. L., Woodward, G., and Hutchinson, C. L. et al.: The ARRL Antenna Book, The American Radio Relay League, Inc. Newington CT, 2-20, 1984.

Jenkins, F. A. and White. H. E.: Fundamentals of Optics, 382-390, McGraw-Hill, New York, 1957.

Kennedy, E.: HAARP Facility Update, in Summary of Presentations, RF Ionospheric Interactions Workshop, Santa Fe, New Mexico, 100-116, 2002.

Kennedy, E.: HAARP Facility Update, Summary of Presentations, RF Interactions Work Shop, Santa Fe, New Mexico, 131-154, 2003.

Liette, A.: 30-40 Megawatt RF Line pulse Generator, Rev. Sci. Instr, 36, 13-15, 1965.

Maxwell, J. C.: A dynamical theory of the electromagnetic field, Phil. Trans. Royal Soc. (London) 155, 459-512, 1864.

Milikh, G.: Efficiency Scaling for Ionospheric ULF/ELF/VLF Generation, Summary of Presentations, RF Ionospheric Interactions Workshop, 489-500, 2002.
Sentman, D., Wuerker, R. F., and McCarrick, M. et al.: Imaging and Spectroscopic Observations of Artificial Airglow Excited by HAARP and HIPAS, RF Ionospheric Interactions Workshop, 632-644, 2002.

Stephan, L. G., Hugrass, W. N., and Jones, I. R.: Two spark gap RF line generator with improved performance, J. Physics E.: Sci. Instr. 15, 992, 1982.

Weibel, E. S.: High Power RF Pulse Generator, Rev. Sci. Instr, 35, 173-74, 1964.

Wong, A. Y., Carroll, J., Dickman, R. et. al.: High-power radiating facility at the HIPAS observatory, Radio Science, 25, 1269-1282, 1990.

Wong, A. Y.: HIPAS Facility Update, in Summary of Presentations, R. F. Interactions Workshoh, Volume I, Santa Fe New Mexico, 107-130, 27-30, 2003. 